\title{
Modeling Multilayer X-Ray Reflectivity Using Genetic Algorithms
}

\author{
M. Sánchez del Río ${ }^{1}$ G. Pareschi ${ }^{2}$, and C. Michetschläger ${ }^{1}$ \\ 1) European Synchrotron Radiation Facility, BP 220, 38043 Grenoble-Cedex, France \\ 2) Osservatorio Astronomico di Brera, Via E. Bianchi 46, I-23807 Merate (Lc), Italy
}

\begin{abstract}
The x-ray reflectivity of a multilayer is a non-linear function of many parameters (materials, layer thickness, density, roughness). Non-linear fitting of experimental data with simulations requires the use of initial values sufficiently close to the optimum value. This is a difficult task when the topology of the space of the variables is highly structured. We apply global optimization methods to fit multilayer reflectivity. Genetic algorithms are stochastic methods based on the model of natural evolution: the improvement of a population along successive generations. A complete set of initial parameters constitutes an individual. The population is a collection of individuals. Each generation is built from the parent generation by applying some operators (selection, crossover, mutation, etc.) on the members of the parent generation. The pressure of selection drives the population to include "good" individuals. For large number of generations, the best individuals will approximate the optimum parameters. Some results on fitting experimental hard x-ray reflectivity data for Ni/C and W/Si multilayers using genetic algorithms are presented. This method can also be applied to design multilayers optimized for a target application.
\end{abstract}

\section{INTRODUCTION}

Many problems in physics can be modeled by using a merit function $f$ which describes the behavior of a system as a function of several parameters $\alpha_{1}, \alpha_{2}, \ldots, \alpha_{n}$. The dependence of $f$ on each parameter can be very complicated and, in most cases, no explicit expression is easily obtained. A common problem is to find the parameters that produce a desired value of $f$. However, this result may be impossible or very difficult to obtain, and usually one looks for solutions close to it. It is convenient to define a figure of merit (FOM) scalar function, which measures the goodness of a solution, or, in other words, the distance from a generic solution $f$ to the ideal solution. The optimization algorithm will look for a minimum (or sometimes a maximum) of the FOM.

In most practical cases, it is not possible to obtain an analytical expression of the FOM that can be minimized using analytical gradient techniques. It is therefore required to compute numerically the FOM. Therefore, the minimum should also be found numerically. An exhaustive exploration of the space of parameters is many 
times unfeasible. Thus, numerical optimization must be applied. Numerical search techniques to find the minimum of the FOM can be classified in two families: local optimization techniques and global optimization methods.

Local optimization techniques [1] look for minimum of FOM closest a given initial point. These methods are useful if: i) the topology of the parameter space is very simple and does not present multiple minima, or ii) we start the optimization with a starting set of parameters sufficiently close to the searched minimum. A particular application of local optimization techniques is the non-linear fitting [2]. Here, the FOM is usually set as the "least squares distance" between experimental data points and model points produced by an algebraic equation that depends on free parameters to be determined. A drawback of local optimization methods is that they are always "attracted" to find the local minimum close to the starting parameters, and the rest of the parameters space is completely ignored. To solve that, one should use global optimization methods. Finding the global minimum is a challenging problem because of the potential existence of many local minima, the high number of dimensions (parameters), uncooperative twists and turns in the topology of the parameters space, and the cost to compute the FOM.

Genetic algorithms (GA) [3,4] are innovative alternative techniques to traditional optimization methods. They use a model similar to the natural process of biological evolution to produce good specialized genotypes through the selection, crossover and mutation operators. They have been applied to fit multilayer reflectivity for neutrons [5] and x-rays [6], and for optimizing the design of interference multilayer filters [7,8].

\section{MOTIVATION AND USE OF GENETIC ALGORITHMS}

The reflectivity of a multilayer is a highly non-linear function of the layer parameters (thickness t, density $\rho$, and roughness $\sigma$ ). Therefore, the FOM (built as least-squares in logarithmic scale between the experimental and modeled reflectivities with data points weighted equally) is also highly non linear on these parameters. To illustrate it, we have simulated the reflectivity of a 10 bilayers of $\mathrm{Ni}\left(\mathrm{t}=45 \AA, \rho=8.876 \mathrm{~g} / \mathrm{cm}^{3}\right)$ and $\mathrm{C}$ $\left(\mathrm{t}=15 \AA, \rho=2.25 \mathrm{~g} / \mathrm{cm}^{3}\right)$. We used a fixed RMS roughness value of $5 \AA$ for all the interfaces. Keeping identical the bilayer composition for all the multilayer stack, we have mapped the FOM as a function of the thickness parameters for both $\mathrm{Ni}$ and $\mathrm{C}$, maintaining fixed all densities and roughness values. The mapping of the figure of merit, i.e., $\mathrm{FOM}\left(\mathrm{t}_{\mathrm{Ni}}, \mathrm{t}_{\mathrm{C}}\right)$ is shown in Fig. 1. The global minimum is located at $(45,15)$ by construction (because we used the simulated reflectivity with these parameters as fake experimental spectrum). If one starts a walk in this space, starting, for example, at $(44,14)$, any local optimization routine will fail in finding the global minimum. This is verified by using the MINUIT fitting program from CERN, demonstrating the difficulty to fit multilayer reflectivities using conventional methods, even in the case of only two free parameters. 
The genetic algorithms work following the following ideas [9]. Suppose we want to find a solution to a problem. We first encode the problem as an artificial chromosome or chromosomes. These artificial chromosomes can be strings of $0 \mathrm{~s}$ and $1 \mathrm{~s}$, parameter lists, or other computer codes, but the important thing to keep in mind is that the genetic machinery will manipulate a finite representation of the solutions, not the solutions themselves. Another thing we must have is a mechanism or procedure for discriminating good solutions from bad solutions. The idea is that something must determine a solution's relative fitness to purpose, and whatever that is will be used by the genetic algorithm to guide the evolution of future generations. We performed that discrimination using our FOM function. We then prepare an initial population of encoded solutions. This initial population is generally created choosing random values of the parameters within their boundary limits. Sometimes one has prior knowledge of possible good solutions, like our a priori initial guess. In such a case, these solutions can be placed as individuals in the initial population. With a population in place, selection and genetic operators can process the population iteratively to create a sequence of populations that hopefully will contain more and more good solutions to our problem as time goes on. There is much variety in the types of operators that are used in GAs, but often i) selection, ii) recombination or crossover and, iii) mutation are used. We have used in our calculations the PGAPack parallel genetic algorithm library by Levine [10]. We have selected it due to the high level of customization (it includes most capabilities desired in a genetic algorithm package), its very easy-to-use interface, its possibility to hybridize, and its efficient FORTRAN binding interface, very useful when the coding of the FOM, as in our case, is done in FORTRAN.
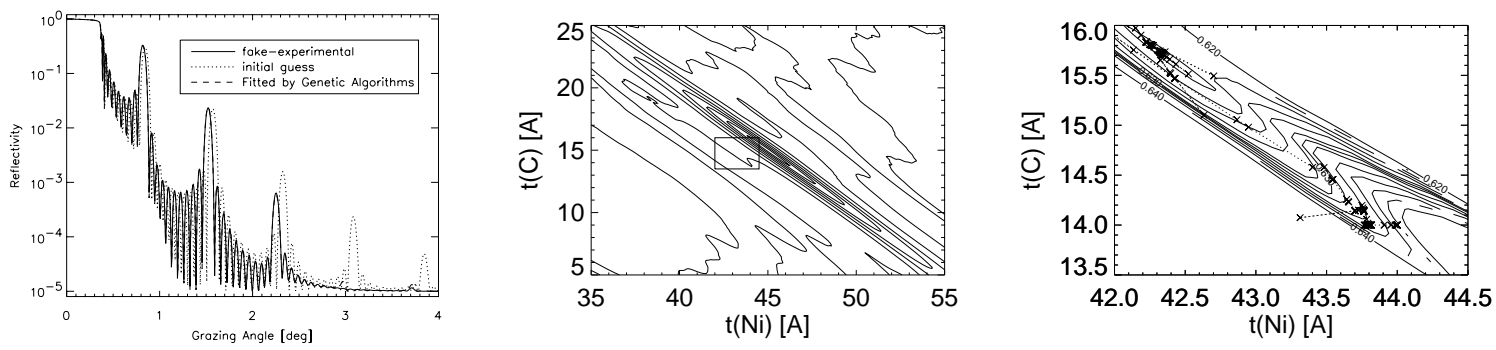

FIGURE 1. Left: the fake experimental data (see text). Dotted line: the starting spectrum (initial guess for the fit at point $(44,14)$ ). Dashed line: the results of the genetic algorithm fit. Center: mapping of FOM as a function of layer thickness using 10 countours in the FOM interval $[0,1]$. Right: a large scale map of the inset, with 10 contours in the FOM interval [0.62,0.64], evidencing a local minimum. The dotted line is the path that the local optimization fitting program MINUIT performs, starting from the point $(44,14)$, to find the local minimum found at $(42.33,15.72)$.

\section{SOME FITS USING GENETIC ALGORITHMS}

In order to test the reliability of our approach we tried to fit a set of real experimental data. In particular we studied the reflectivity as a function of the angle of incidence for a number of multilayer samples deposited onto substrates of different kind. All the x-ray test were performed at $8 \mathrm{keV}$ photon energy making use of two 
different $x$-ray facilities (the BM5 facility at ESRF and a BEDE Scient. D1 System diffractometer installed at the Brera Astronomic Observatory, Italy). In both cases the primary beam was characterized by a high degree of monochromatization $\left(\Delta \mathrm{E} / \mathrm{E} \sim 10^{-4}\right)$ and a very low angular divergence ( $<12$ arcsec). The multilayer reflectivity is modeled with the standard recursive method [11], Some fitting results obtained using genetic algorithms include three different $\mathrm{Ni} / \mathrm{C}$ multilayer samples realized by ionbean sputtering at Ce.Te.V. (Center for Vacuum Technology Carsoli, Italy). These deposition have been done as a part of a project carried out by the Brera Astronomical Observatory having the aim to set-up a method for the production of x-ray Wolter I astronomical optics based on multilayer coating [12]. The results are presented in Fig. 2 .
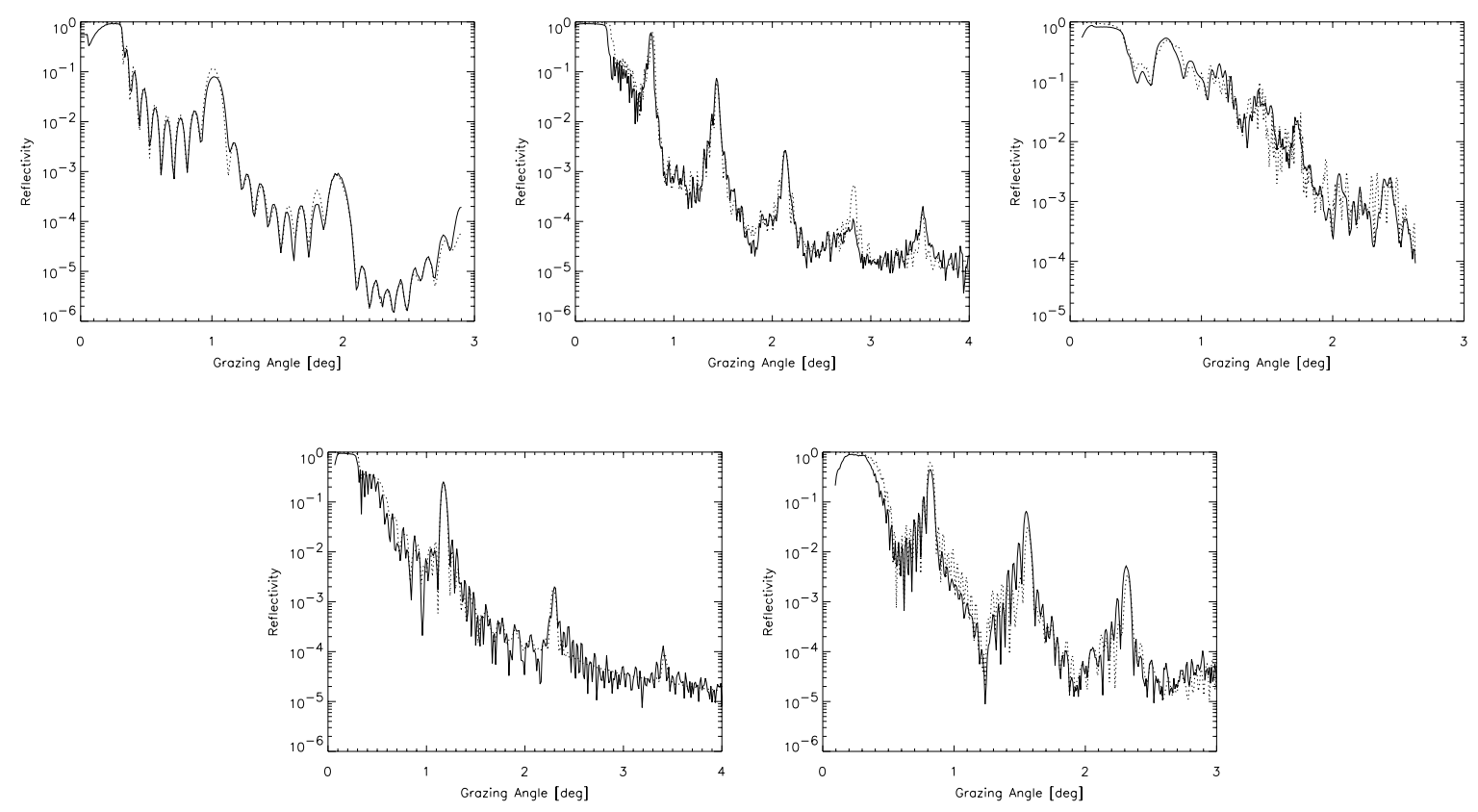

FIGURE 2. Fits of reflectivity versus incidence angle (in degrees) for a number of samples. The continuous line is the experiment and the dotted line is the fit. i) Sample with, 9 bilayers $\mathrm{Ni} / \mathrm{C}$ on $\mathrm{SiO}_{2}$ substrate, $\Gamma \sim 0.45$ d 46 ̊. Fitting up to 59 parameters. ii) Sample with 19 bilayers Ni/C on Ni substrate, $\Gamma \sim 0.73 \mathrm{~d} \sim 64 \AA$ A. Fitting up to 122 parameters. iii) Sample with 92 graded bilayers of W/Si on $\mathrm{Si}$ substrate, $\Gamma \sim 0.47$ d 30-100 ̊. Fitting up to 554 parameters. iv) Sample with 19 bilayers $\mathrm{C} / \mathrm{Ni}$ on $\mathrm{SiO}_{2}$ substrate. $\Gamma \sim 0.4$ d 40 A. Fitting up to 119 parameters. v) Sample with 19 bilayers $\mathrm{C} / \mathrm{Ni}$ on $\mathrm{SiO}_{2}$ substrate. $\Gamma \sim 0.65 \mathrm{~d} \sim 58 \AA$. Fitting up to 112 parameters. $\Gamma$ is the ratio between the thickness of the heavy element layer and the bilayer thickness $d$.

In conclusion, we show that fitting multilayer reflectivity to a model is a very difficult task if one wants to use conventional (local) fitting algorithms. We have applied evolutionary computing (genetic algorithms) to perform this type of fitting with very promising results. We are now optimizing our method by using hybridization (coupling genetic algorithms with local optimization techniques) in order to get better convergence when the algorithm finds a local minimum. We are currently applying this technique to optimize the design of multilayers for focusing $\mathrm{x}$ ray telescopes. 


\section{ACKNOWLEDGMENTS}

We thank E. Ziegler (ESRF), C. Ferrero (ESRF) and O. Citterio (OAB) for helpful discussions. We are also grateful to the ESRF BM5 staff for assistance during the experiment. The multilayer samples were produced at Ce.Te.V. (Carsoli, Italy).

\section{REFERENCES}

1. E. Polak, Computational Methods in Optimization, Academic Press, New York, 1971.

2. W. H. Press, B. P. Flannery, S. A. Teukolsky and W. T. Vetterling, Numerical Recipes. The art of scientific computing, Cambridge University Press, Cambridge, 1989.

3. J. H. Holland, Adaptation in Natural and Artificial Systems, University of Michigan Press, Ann Arbor, 1975.

4. D. Goldberg, Genetic Algorithms in Search, Optimization and Machine Learning, Addison-Wesley, Reading, MA, 1989.

5. V. O. DeHaan and G. G. Drijkoninger, Physica B 53, 24, 1994

6. A. D. Dane, A. Veldhuis, D. K. G. de Boer, A. J. G. Leenaers and L. M. C. Bruydens, Physica B 253, 254-268, 1998

7. T. Eisenhammer, M. Lazarov, M. Leutbecher, U. Schöffel and R. Sizmann, Applied Optics 32, 6310-6315, 1993

8. S. Martin, J. Rivory and M. Schoenauer, Applied Optics 34, 2247-2254, 1995

9. D.E. Goldberg, Genetic and evolutionary algorithms in the real world, Illinois Genetic Algorithms Laboratory. Internal Report 99013, ftp://ftp-illigal.ge.uiuc.edu/pub/papers/IlliGALs/99013.ps.Z, 1999.

10. D. Levine, Users Guide to the PGAPack Parallel Genetic Algorithm Library, Argonne National Laboratory Internal Report http://www-unix.mcs.anl.gov/ levine/PGAPACK/index.html , 1996.

11. K. D. Joensen, P. Voutov, A. Szentgyorgyi, J. Roll, P. Gorenstein, P. Hoghoj, and F. E. Christensen, Appl. Opt. 34, pp. 7935-7944, 1995.

12. O. Citterio, P. Cerutti, F. Mazzoleni, G. Pareschi, E. Poretti, P. Lagana, A. Mengali, C. Misiano, and F. Pozzilli, SPIE Proc, 3766, 1999 (in press). 